\title{
GMR biosensor arrays: A system perspective
}

\author{
D.A. Hall ${ }^{\mathrm{a}, 1}$, R.S. Gaster ${ }^{\mathrm{b}, \mathrm{c}, 1}$, T. Lin ${ }^{\mathrm{a}}$, S.J. Osterfeld ${ }^{\mathrm{d}}$, S. Han ${ }^{\mathrm{e}}$, B. Murmann ${ }^{\mathrm{a}}$, S.X. Wanga,f,* \\ a Department of Electrical Engineering, Stanford University, Stanford, CA 94305, USA \\ ${ }^{\mathrm{b}}$ Department of Bioengineering, Stanford University, Stanford, CA 94305, USA \\ ${ }^{c}$ Medical Scientist Training Program, Stanford University School of Medicine, Stanford, CA 94305, USA \\ d MagArray, Inc., Sunnyvale, CA 94089, USA \\ e IBM T. J. Watson Research Center, Yorktown Heights, NY 10598, USA \\ ${ }^{\mathrm{f}}$ Department of Materials Science and Engineering, Stanford University, Stanford, CA 94305, USA
}

\section{A R T I C L E I N F O}

Article history:

Received 26 September 2009

Received in revised form 1 December 2009

Accepted 31 January 2010

Available online 6 February 2010

\section{Keywords:}

Magnetic biosensor

GMR biosensor

Spin-valve biosensor

Multiplexing spin-valves

\begin{abstract}
A B S T R A C T
Giant magnetoresistive biosensors are becoming more prevalent for sensitive, quantifiable biomolecular detection. However, in order for magnetic biosensing to become competitive with current optical protein microarray technology, there is a need to increase the number of sensors while maintaining the high sensitivity and fast readout time characteristic of smaller arrays (1-8 sensors). In this paper, we present a circuit architecture scalable for larger sensor arrays (64 individually addressable sensors) while maintaining a high readout rate (scanning the entire array in less than $4 \mathrm{~s}$ ). The system utilizes both time domain multiplexing and frequency domain multiplexing in order to achieve this scan rate. For the implementation, we propose a new circuit architecture that does not use a classical Wheatstone bridge to measure the small change in resistance of the sensor. Instead, an architecture designed around a transimpedance amplifier is employed. A detailed analysis of this architecture including the noise, distortion, and potential sources of errors is presented, followed by a global optimization strategy for the entire system comprising the magnetic tags, sensors, and interface electronics. To demonstrate the sensitivity, quantifiable detection of two blindly spiked samples of unknown concentrations has been performed at concentrations below the limit of detection for the enzyme-linked immunosorbent assay. Lastly, the multiplexing capability and reproducibility of the system was demonstrated by simultaneously monitoring sensors functionalized with three unique proteins at different concentrations in real-time.
\end{abstract}

(c) 2010 Elsevier B.V. All rights reserved.

\section{Introduction}

Giant magnetoresistance (GMR) based biosensors capable of highly sensitive detection are poised to become a dominant player in the vast world of biosensors. The rapid development partially came about from leveraging existing sensing technology utilized in hard disk drives. Spin-valves exhibit the GMR effect, a quantum mechanical phenomenon wherein a change in the local magnetic field induces a change in resistance due to spin-dependent scattering in elaborately engineered magnetic multilayer or sandwich films. In 1998, Baselt et al. (1998) were the first to demonstrate using GMR sensors as biosensors and several groups have continued the research and development of magnetic biosensing technology (Ferreira et al., 2003; Rife et al., 2003; Reiss et al., 2005;

\footnotetext{
* Corresponding author at: Department of Materials Science and Engineering, Stanford University, 476 Lomita Mall, McCullough Bldg R351, Stanford, CA 94305, USA.

E-mail address: sxwang@stanford.edu (S.X. Wang).

${ }^{1}$ These authors contributed equally to this work.
}

de Boer et al., 2007; Xu et al., 2008; Osterfeld et al., 2008; Koets et al., 2009; Edelstein et al., 2000).

GMR biosensors for protein assays operate in a similar fashion to the enzyme-linked immunosorbent assay (ELISA) where a capture antibody is immobilized on the surface of the sensor and the analyte of interest selectively binds to the antibody. The assay is completed by introducing a detection antibody labeled with an externally observable tag. Unlike ELISA where the tag is typically a fluorescent molecule, GMR biosensors rely on a magnetic tag. The sensors detect the stray field from the magnetic tag to infer the number of captured analytes.

Biosensors utilizing magnetic tags offer several key advantages over other sensing modalities. First, the biological samples (blood, urine, serum, etc.) naturally lack any detectable magnetic content, providing a sensing platform with a very low background level and thus lower detection limit of analytes. Second, the sensors can be arrayed and multiplexed to perform analysis on a panel of proteins or nucleic acids in a single assay. Lastly, the sensors can be manufactured cheaply, in mass quantities, to be deployed in a one-time use disposable format. For these reasons, magnetic biosensors are an attractive and competitive alternative to optical techniques. 
Increasingly larger panels of proteins are being investigated by both researchers and physicians for minute expression changes in order to diagnose a wide variety of diseases. However, most of the research to date has focused on theory and proof of concept work (Ferreira et al., 2005; de Boer et al., 2007; Li and Wang, 2003) or point of care implementations using a small array of sensors (Edelstein et al., 2000; Dittmer et al., 2008). Furthermore, there has been little attention to the readout time of the arrays. In this paper, we present a circuit architecture that is highly sensitive and scalable for larger sensor arrays with a pointed effort to decrease the scan time. Methods for multiplexing the sensors are introduced which substantially decrease the readout time of the array, allowing us to monitor the sensors in real-time (Gaster et al., 2009; Osterfeld et al., 2008). We begin by presenting the sensors and the magnetic nanoparticle (MNP) tags, followed by a comprehensive analysis of the sensor interface including noise and distortion. We demonstrate biological detection of blindly spiked samples at concentrations that are undetectable by ELISA as well as a multiprotein assay. To conclude, we present a comparison of the system described here to prior art in GMR biosensor systems.

\section{Materials and methods}

\subsection{Sensors}

A custom sensor die containing an $8 \times 8$ of spin-valve sensors (Fig. 1a) was used in all experiments. The die is $1 \mathrm{~cm} \times 1.2 \mathrm{~cm}$ with a sensor to sensor pitch of $300 \mu \mathrm{m}$. Each sensor occupies an area of $90 \mu \mathrm{m}$ by $90 \mu \mathrm{m}$ and is constructed by combining parallel sets of GMR stripes (48 in total) in series to set the coverage area independently of the resistance (nominally $2.5 \mathrm{k} \Omega$ ). The spin-valves were deposited on an $\mathrm{Si} / \mathrm{SiO}_{2}$ substrate with the following GMR struc- ture (all thicknesses in nm): Ta (3), seed layer (4), PtMn (15), CoFe (2), Ru (0.85), CoFe (2), Cu (2.3), CoFe (2), Cu (1), Ta (4) (Osterfeld et al., 2008). The entire structure, including the leads, was passivated with $\mathrm{SiO}_{2}(10 \mathrm{~nm}), \mathrm{Si}_{3} \mathrm{~N}_{4}(20 \mathrm{~nm})$, and $\mathrm{SiO}_{2}(10 \mathrm{~nm})$ to isolate the sensors from the various biological and chemical solutions that are present in the reaction well. In this design, the maximum voltage that can be applied to the sensor is limited to $0.5 \mathrm{~V}$ to avoid breakdown of the thin passivation layer. The sensor die was glued and wirebonded to an 84 pin ceramic package with an open well (Fig. 1b) or a microfluidic chamber on top.

The transfer curve of the spin-valve sensor is shown in Fig. 1c and was measured by sweeping the field strength along the hard axis (energetically unfavorable direction of magnetization) of the sensing layer without any other magnetic biasing. The sensor has a minimum resistance of $2190 \Omega$ in the antiparallel state and a maximum resistance of $2465 \Omega$ in the parallel state, corresponding to a magnetoresistance ratio (MR) of $12 \%$. The sensitivity of the sensor was calculated by differentiating the transfer curve (Fig. 1d), and is maximum when no field is applied, tapering off as the field strength is increased. The non-linearity of the sensor is also shown in Fig. 1d. Non-linearity creates harmonic distortion in the output spectrum, an important non-ideality to consider when frequency domain multiplexing is utilized. Lastly, the flicker noise corner frequency (the frequency where the flicker noise intersects the thermal noise) measured was $900 \mathrm{~Hz}, 2.25 \mathrm{kHz}$, and $10 \mathrm{kHz}$ for currents of $100 \mu \mathrm{A}, 250 \mu \mathrm{A}$, and $500 \mu \mathrm{A}$ respectively (Supplementary Fig. 1). These high corner frequencies typically cause flicker noise to be the limiting factor in the sensitivity of the entire system (de Boer et al., 2007), particularly if the signal cannot be modulated to a higher frequency. A more detailed analysis of how these affect the overall system will be presented when considering the entire signal path. (a)
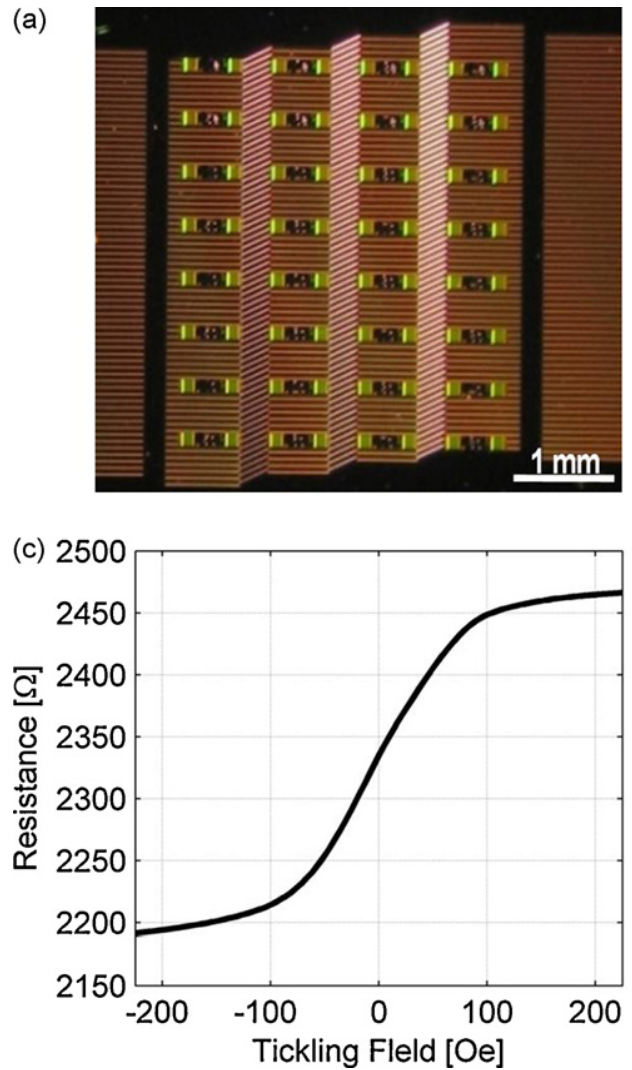

(b)

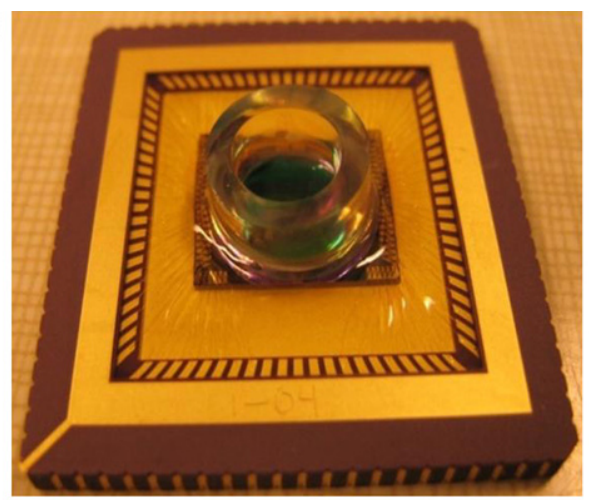

(d)

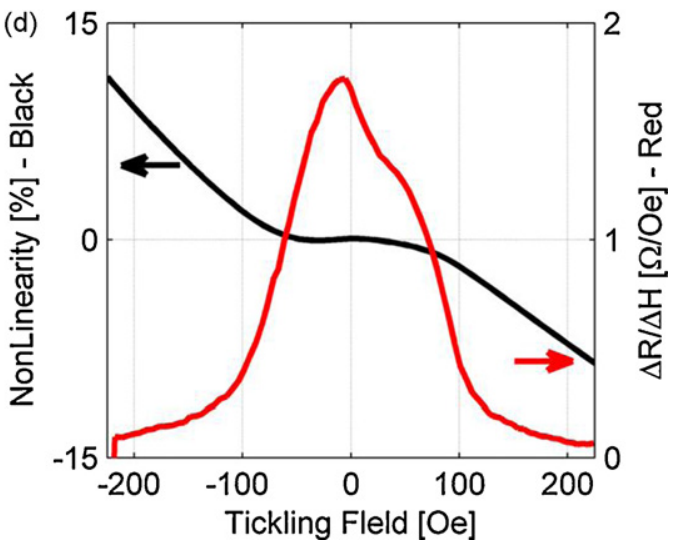

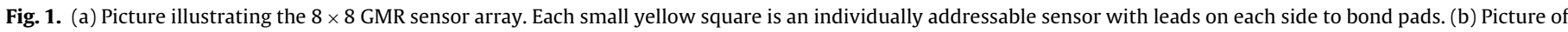

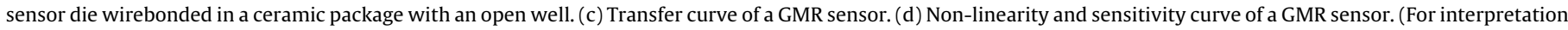
of the references to color in this figure legend, the reader is referred to the web version of the article.) 
Typically, GMR sensors require two magnetic fields: a biasing field along the easy axis (energetically favorable direction of magnetization) to force the free layer into a single domain state and a tickling field along the hard axis to magnetize the superparamagnetic tags and modulate the signal to a higher frequency away from the flicker noise of the sensor. However, due to the geometry of the sensors, there is sufficient shape anisotropy such that the external magnetic bias field can be removed. Many groups (de Boer et al., 2007; Koets et al., 2009; Dittmer et al., 2008) have opted to generate the magnetic tickling field on the same die as the sensors. While there are many advantages to integrating the coil, namely precise alignment (due to photolithography), miniaturization, high frequency operation, and the ability to magnetically attract the particles to the sensor surface; they suffer from poor field uniformity (de Boer et al., 2007). The field uniformity is crucial in achieving reproducible results across different sensors and different dies, particularly at low analyte concentrations. As a result of this issue, we instead chose to use an external Helmholtz coil from Oersted Technology. This coil is capable of generating up to 100 Oe fields while being driven by a Kepco BOP 50-4 M power amplifier, but is limited to frequencies less than $500 \mathrm{~Hz}$.

\subsection{Magnetic nanoparticles}

The MACS magnetic tags used in all experiments are manufactured by Miltenyi Biotec. These nanoparticles are clusters composed of monodisperse $13 \mathrm{~nm} \mathrm{Fe} \mathrm{O}_{3}$ nanoparticles embedded in a dextran shell ( $50 \mathrm{~nm}$ in diameter) functionalized with streptavidin. They are superparamagnetic, meaning that they exhibit little or no magnetic moment in the absence of an external magnetic field. Even though MACS MNP tags are very small compared to many larger particles used in the literature, they are detectable because of the ultrathin passivation and high sensitivity of the sensors (Osterfeld et al., 2008). Another benefit of using small tags is that the unbound tags do not need to be removed from the assay (by washing) because they remain in solution and are not in close enough proximity to the sensor to be detected.

\subsection{Operating principles}

Using the model proposed by Li and Wang (2003), we derived a closed form equation predicting the change in resistance due to the presence of a single MNP. This model incorporates simplifying assumptions in order to make the calculations tractable without having to resort to finite element modeling simulations. Namely, the particle can be approximated as a dipole positioned in the center of the sensor. While in reality the response will be dependent on the location of the magnetic particle, on average, this is a reasonable approximation (Ferreira et al., 2005). The second assumption is that the individual stripes of the sensor are far enough away such that the particle only interacts with one stripe in the sensor, in addition, particle to particle interactions can be neglected. Under these assumptions, we calculated that each MNP induces a $4.6 \mu \Omega$ resistance change.

$\frac{\Delta R}{\operatorname{tag}}=-\frac{8 m_{s}\left(\operatorname{coth}\left(\left(m_{s} H_{a}\right) / k_{B} T\right)-\left(k_{B} T /\left(m_{s} H_{a}\right)\right)\right)}{\left(w^{2}+4 d^{2}\right) \sqrt{l^{2}+w^{2}+4 d^{2}}} \cdot \frac{\partial R_{S}\left(H_{a}\right)}{\partial H}$

Eq. (1) shows the result originally derived by Li and Wang (2003) multiplied by the sensitivity function of the spin-valve. There are additional fractional coefficients (not shown) which result from calculating the resistance change on a single stripe and subsequently referring it to the entire sensor (composed of many stripes). By applying the largest voltage that does not cause the sensor passivation to breakdown $(0.5 \mathrm{~V})$, the calculated current change per particle is $370 \mathrm{fA}$.
We used a double modulation scheme to maximize the signal to noise ratio (SNR) by modulating both the applied magnetic field (at a frequency $f_{f}$ ) and the voltage applied to the sensor (at a frequency $f_{c}$ ) (de Boer et al., 2007; Han et al., 2007). Modulating the excitation voltage moves the signal to a higher frequency, away from the flicker noise of the interface electronics, while modulating the magnetic field reduces the flicker noise of the sensor, eliminating it entirely if the modulation frequency is above the flicker noise corner. Combined, both of these modulations cause the sensor to operate as a mixer. The output spectrum has three primary tones, a carrier tone at $f_{c}$, and two side tones at $f_{c} \pm f_{f}$ separating the magnetoresistive component (the portion of resistance that responds to the magnetic tags, typically only a few percent of the total resistance) and the non-magnetoresistive component of the sensor. Additionally, there is a parasitic tone from inductive coupling picked up by the leads to the sensor at $f_{f}$.

\subsection{Implementation details}

Historically, a Wheatstone bridge has been ubiquitous for high precision resistance measurements. For this application, the bridge is typically constructed of two spin-valve sensors and two programmable resistors. One of the sensors is biologically active and the other is a reference sensor, magnetically isolated but physically near the active sensor such that it experiences the same sensing environment (such as temperature changes, magnetic bias and tickling field gradients). The differential output of the bridge cancels the common mode signal experienced by both the active and reference sensor. A high-gain instrumentation amplifier (IA) amplifies the small differential output of the bridge caused by the MNPs.

However, balancing the bridge requires tuning the two programmable resistors to compensate for mismatch in the sensor resistances due to process variations. If the bridge is not balanced, the offset can cause the high-gain differential amplifier to saturate. For small arrays, this tuning step is not problematic; however, it becomes cumbersome for larger arrays, particularly if a rapid scan rate is needed. Furthermore, a bridge interface is not easily multiplexed, limiting its utility in large arrays. To address these problems, we propose an architecture that uses a transimpedance amplifier (TIA) to measure the small resistance changes (Fig. 2 and Supplementary Fig. 2). In this circuit, an excitation voltage is applied to the sensor and the resulting current is converted back to a voltage by the TIA. Once digitized, this voltage change is proportional to the resistance change.

One significant advantage that magnetic biosensors have over some competing technologies is the ability to look at binding events in real-time (Osterfeld et al., 2008) compared to two point, preand post-experiment, measurements. As with many biological processes, these events occur at relatively slow rates (on the order of hundreds to thousands of milliseconds). As the size of the sensor array increases, it becomes more difficult to scan the entire array while maintaining a rapid point to point scan time resolution. To decrease the time it takes to readout the array, we exploit both frequency domain multiplexing (FDM) and time domain multiplexing (TDM). The time domain multiplexing is implemented by the switches at the input controlled by non-overlapping clock phases, as shown in Fig. 2. The frequency domain multiplexing is performed by exciting the sensors with different carrier frequencies and summing the currents at the virtual ground of the TIA. The response of an individual sensor is extracted after digitization in the frequency domain by spectral analysis.

A shortcoming of not using integrated coils (which could accommodate tickling fields of higher frequencies) is that the frequency range of the tickling field is limited by the external coil, typically a few $\mathrm{kHz}$ or less. Since the frequency separation between the carrier tone and side tone is determined by the coil frequency, it is very 


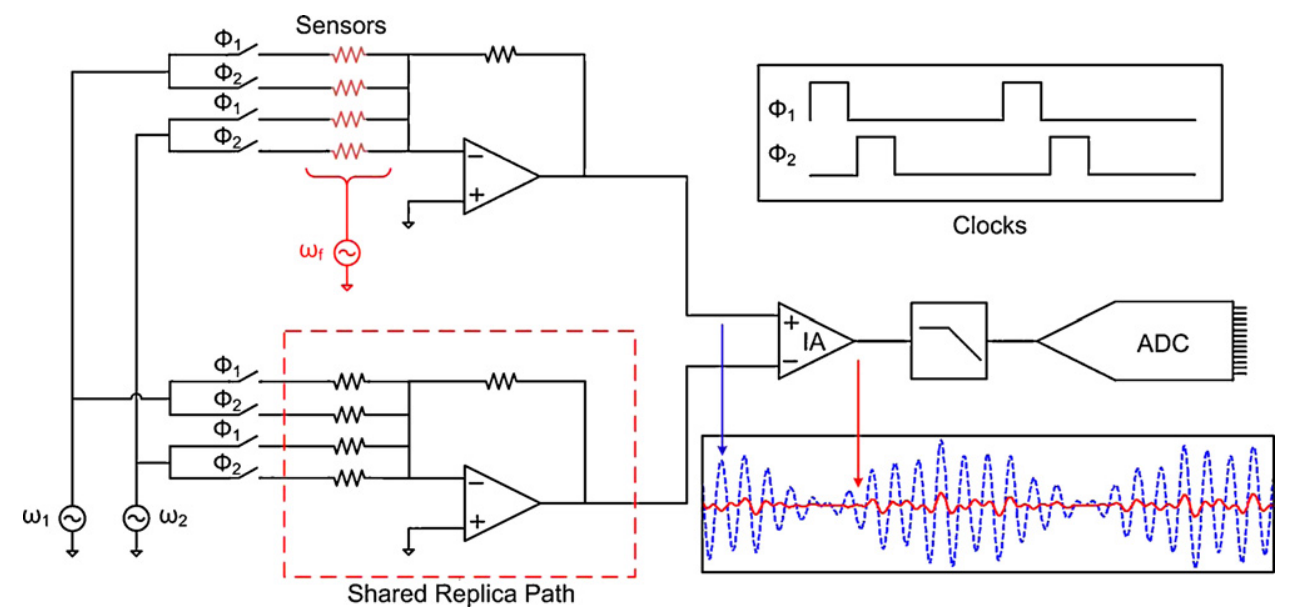

Fig. 2. System architecture based on a transimpedance amplifier with a shared replica path for carrier suppression.

difficult to filter out the carrier tone. For example, a bandpass filter centered at $700 \mathrm{~Hz}$ with a $10 \mathrm{~Hz}$ bandwidth requires a quality factor $(Q)$ of 70 , a filter that is difficult to realize in hardware. Furthermore, since the MR is only a few percent of the resistance, the carrier tone will typically be at least $30 \mathrm{~dB}$ larger than the side tone. This requires the analog front end to have a large dynamic range and the resolution requirement of the ADC becomes prohibitive to digitize the signals directly. To address this, we added a replica path that is used to suppress the carrier tone. In the replica path, the sensors are replaced with either fixed or adjustable resistors, so the spectrum of this path contains only the carrier tones and not the side tones (the signals of interest). An IA subtracts the carrier tones from the signal and provides additional amplification before the signal is digitized.

A National Instruments NI-6281 data acquisition card digitizes the signals from the amplifier board. This card has an 18-bit analog to digital converter (ADC) and easily interfaces with LabVIEW. The different frequency excitation sources are generated with an NI-6133, which has a 16-bit digital to analog converter (DAC). Custom LabVIEW software (Supplementary Figs. 3-5) gathers samples from each set of sensors, applies a window function, and performs a Fourier transform to extract the amplitudes of the different tones of interest. The change in amplitude of the side tones over time (a spectrogram) is proportional to the change in MR of the spin-valve. The sampling rate of the ADC is limited by the settling time of the multiplexer that precedes it and the number of samples is chosen in order to achieve a sufficiently small effective noise bandwidth (ENBW). The LabVIEW software also applies all the custom correction algorithms and displays the collected data to the end-user (Hall et al., in press).

One notable advantage of the TIA based topology is instead of acquiring the difference signal between the active and reference sensor, both signals are acquired independently allowing the reference to be chosen after the test has been performed. The common mode signal can be used to determine information about the sensors and the sensing environment such as the magnetic field non-uniformity and the relative temperature change. Furthermore, many complex referencing schemes are easily implemented. For example, if there are multiple reference sensors in the array, the reference for a particular biologically active sensor can be chosen based on spatial locality, an average of all the references, or the reference with the closest resistance or MR. Referencing in software provides an extra degree of flexibility not found in dedicated hardware systems.

For a sensor interface, the circuit should be transparent to the sensor, following the adage "Do No Harm" (Makinwa et al., 2007). For one sensor, assuming that the amplifier is operating beyond the
$1 / f$ noise corner, the input referred noise density was calculated to be $4.9 \mathrm{pA} / \sqrt{ } \mathrm{Hz}$. When multiple sensors are added in parallel to the summing node, the input referred noise increases to $7.1 \mathrm{pA} / \sqrt{ } \mathrm{Hz}$, $11.5 \mathrm{pA} / \sqrt{ } \mathrm{Hz}$, and $20.7 \mathrm{pA} / \sqrt{ } \mathrm{Hz}$ for 2,4 , and 8 sensors respectively. Alone, the sensor has a thermal noise density of $2.6 \mathrm{pA} / \sqrt{ } \mathrm{Hz}$; however, at the current and frequency of operation, the sensor is still well within the $1 / f$ regime. Taking into account the $1 / f$ noise (at $215 \mathrm{~Hz}$ ), the sensor has a total current noise density of $12.9 \mathrm{pA} / \sqrt{ } \mathrm{Hz}$. In a $1 \mathrm{~Hz}$ bandwidth, theoretically as few as 70 magnetic nanoparticles can be detected with an SNR of 2 (Eq. (2)). The bandwidth that the noise is integrated over is a design parameter chosen based on the desired output rate which trades off with the minimum detectable signal.

Min. number tags $=\frac{S N R_{\text {desired }} \sqrt{N_{t h}^{2}+N_{1 / f}^{2}} \cdot \sqrt{B W}}{V /(\Delta R / \operatorname{tag})}$

Inherent non-linearity in the spin-valve transfer curve leads to higher order harmonics in the output spectrum and further imposes strict requirements on the amplifier linearity due to intermodulation distortion (IMD) products. Furthermore, the frequencies of the carrier tones and the coil must be chosen carefully to prevent any harmonics or IMD tones from overlapping other carrier tones or side tones. As the tickling field strength is increased (as shown in Fig. 1d), the non-linearity also increases, a result that is critical in the system optimization.

The partitioning of sensors between frequency domain multiplexing and time domain multiplexing is an optimization problem that requires balancing low noise, low distortion, reasonable hardware resources, and quick readout time. In the absence of noise and distortion, the optimum partitioning is a linear tradeoff between the amount of hardware (the number of excitation sources) and the readout time. Yet, when noise and distortion are considered, the partitioning tends to shift to a more conservative approach of time domain multiplexing more sensors. As previously shown, the input referred noise increases as more sensors are frequency domain multiplexed. However, the noise is often limited by the flicker noise of the device so the additive white noise does not degrade the overall performance significantly. The distortion, on the other hand, is troubling because as the number of sensors multiplexed in the frequency domain increases, the IMD becomes problematic due to the large number of tones in the spectrum. In our current implementation with 64 sensors, we chose to FDM either 2 or 4 tones and TDM 4 or 2 respectively to meet our design objective of a 4-s readout time.

There are several potential sources of error in the circuit shown in Fig. 2; however, it is pertinent to note that the absolute accuracy is not as important as the precision and repeatability. Errors such as 


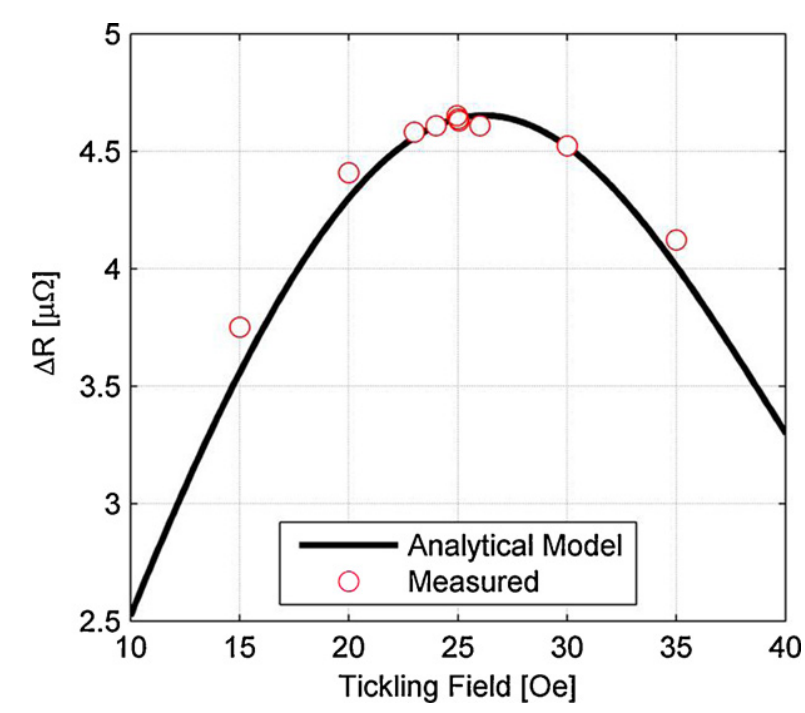

Fig. 3. Tickling field versus resistance change per magnetic nanoparticle tag.

lead resistance (from the amplifier board to the coil and back), excitation amplitude, amplifier offset, and finite gain of the TIA result in a reproducible offset in the amplitude of the side tone. The offset is normalized away because the signal of interest is the time-varying component of the side tone amplitude, not the absolute value. Gain errors such as the IA and TIA gain (set by the feedback resistor) are remedied by using a digital calibration routine (Hall et al., in press).

A bridge interface naturally cancels out the temperature dependence of the sensor as long as the thick oxide passivation does not thermally isolate the reference sensor. Since spin-valves often have fairly large temperature coefficients (hundreds to thousands of $\left.\mathrm{PPM} /{ }^{\circ} \mathrm{C}\right)($ Daughton and Chen, 1993; Lenssen et al., 2000) and experience large temperature swings from the chemical and biological solutions placed on the sensors (up to $30^{\circ} \mathrm{C}$ swings are possible), the temperature signals can easily be much larger than the small signal from MNPs being sensed. To correct for this temperature dependence in the TIA architecture, we designed an algorithm that uses the carrier tone to monitor the relative temperature change and applies a correction factor to the side tones (Hall et al., in press).

\subsection{SNR optimization}

Given the heterogeneity of the system, comprised of magnetic tags, sensors, and electronics, there are dozens of design parameters and complex tradeoffs with interleaved relationships. However, these tradeoffs can be reduced to two primary design parameters: the amplitude of the tickling field and the amplitude of the bias field because both affect the moment of the superparamagnetic nanoparticle and the sensitivity of the sensor. As mentioned previously, with our $750 \mathrm{~nm}$ sensor widths, the shape anisotropy is sufficient for biasing and the external bias field can be removed. The tickling field amplitude required to maximize the signal per particle can be determined by multiplying the sensitivity curve of the sensor (Fig. 1d) and the transfer curve of the magnetic nanoparticles. The sensor is most sensitive with no applied field; however, the superparamagnetic particles have no moment without an external field. As the external field is increased, the increasing moment of the magnetic particles compensates for the loss of sensitivity. As the field is increased further though, the non-linearity of the sensor dominates and increasing the field further reduces the measurable signal. Fig. 3 shows the experimental results of changing the tickling field strength overlaid with the optimum computed from the model proposed in Li and Wang (2003). There is good agreement between the two even with the simplifications in the model. The optimum tickling field for this combination of MACS MNP and sensor is 25 Oe.

\section{Results and discussion}

For all protein detection experiments, we used a sandwich assay wherein the protein of interest is sandwiched between two antibodies, one covalently bound to the sensor surface (known as the capture antibody) and the other, added in solution, functionalized with biotin (known as the detection antibody). Therefore, upon subsequent addition of MNP tags labeled with streptavidin, the MNPs will bind to the detection antibody via streptavidin-biotin interaction. In addition, as a negative control to monitor non-specific binding in the system, several sensors were functionalized with bovine serum albumin (BSA) instead of a capture antibody.

In order to investigate the sensitivity of our GMR biosensor arrays, a series of quantitative experiments monitoring carcinoembryonic antigen (CEA) were conducted. To begin, a calibration curve was generated via serial dilution of known concentrations of human CEA spiked into $0.1 \%$ BSA in saline. From the calibration curve shown in Fig. 4, it is apparent that detection of CEA is possible down to the fM-level without any biological amplification. At the lower limit of detection ( $5 \mathrm{fM}$ ), we estimate that there are approximately 2200 surface bound MNPs. Since our calibration curve is linear (on a log-log plot) all the way down to this concentration regime, highly sensitive and quantitative protein detection is possible.

While initially it seems natural to report the output voltage difference between the final amplitude and the initial amplitude of the side tone, the meaning of this quantity is strongly dependent on the test parameters. For example, the result is scaled with the excitation voltage making comparisons between different measurement setups or implementations difficult. A better metric is the change in MR of the sensor, a property which is fundamentally changing and un-scaled by the current or voltage applied to the sensor. All of the results presented here are changes in MR normalized to the initial MR in parts per million (PPM).

It is important to note that the MNPs we use are colloidally stable and therefore, they do not settle over time on the sensor surface. To prove this, one can observe that the control sensors, coated with BSA or a non-complementary antibody, produced negligible signals throughout the entirety of the experiment. The colloidal stability as well as the blocking buffer used dramatically reduces any nonspecific binding in the system and has allowed us to push down the lower limit of detection to the fM-level. In addition, there is little advantage to magnetically attracting the MNP tags to the sensor surface in our case, contrary to many reports in the literature (Koets et al., 2009; Martins et al., 2009). Although kinetically the binding of MNP tags to the detection antibody will increase the rate of diffusion, it will also dramatically increase non-specific binding in the assay, undesirably raising the lower limit of detection. Furthermore, the total assay time is often more limited by the analyte incubation time than the MNP tag binding time.

To elucidate the accuracy of our protein detection platform in serum samples, we conducted a blind study on two samples of human CEA spiked into mouse serum at concentrations unknown to our group. For statistical reproducibility, each blind sample was monitored by 12 unique sensors functionalized with CEA monoclonal capture antibody. Using a calibration curve, the average signal from each unknown sample after 20 min MNP incubation was converted into a molar concentration. We measured mouse serum sample A to contain $66 \mathrm{fM}$ CEA and mouse serum sample $B$ to contain 6.9 fM CEA. Mouse serum sample A was revealed to have been spiked with $75 \mathrm{fM}$ CEA while mouse serum sample $B$ contained $7.5 \mathrm{fM} \mathrm{CEA}$, a $12 \%$ and $8 \%$ deviation from the experimentally observed values. In addition, both samples were run on an ELISA for comparison, however, no signals were detectable as the protein content in these samples was orders of magnitude lower than the lower limit of detection for ELISA. Therefore, with our 


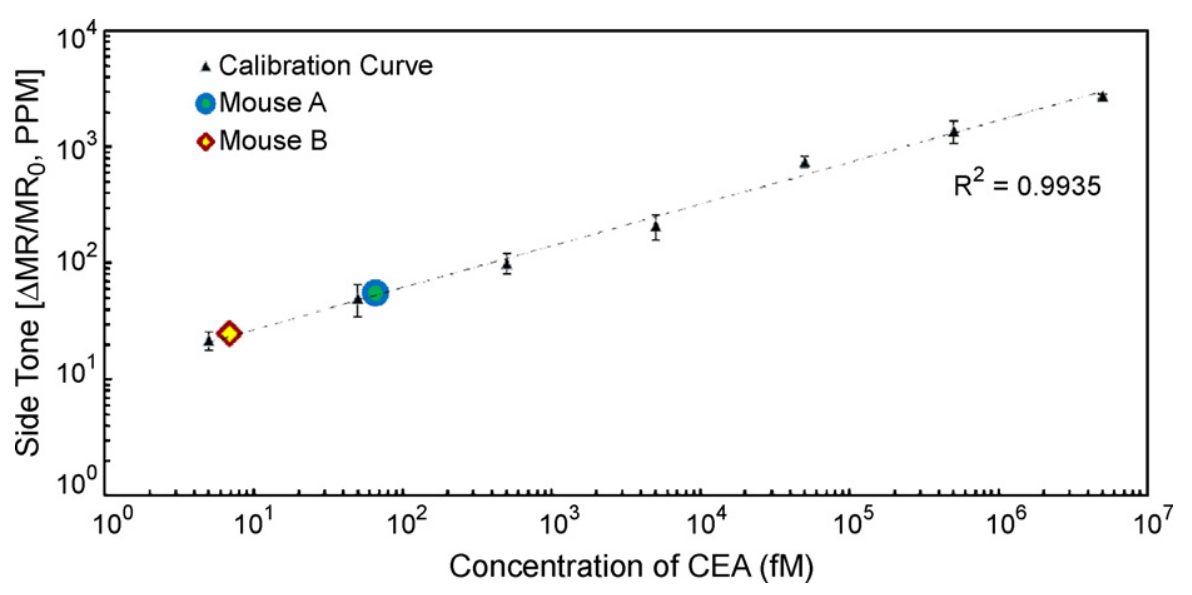

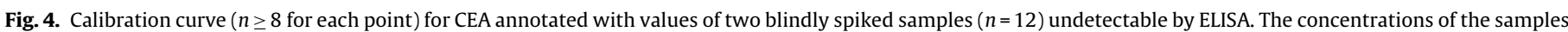
were accurate to within $15 \%$. Error bars are \pm 1 standard deviation.

protein detection platform, we are capable of monitoring protein expression levels at concentrations well below the most prevalent technologies.

The multiplex capabilities of our GMR biosensor arrays are also vast. Here, we show the ability to distinguish a multiplicity of proteins present at a wide variety of concentrations in a single reaction well. In Fig. 5, a time elapsed 3D representation of the signal from each sensor in the array was plotted (see Supplementary Fig. 6 for the 2D plot of the data and Supplementary Fig. 7 for the online movie). At time zero, there is no signal. At three and a half minutes, the MNP tags are actively binding to the detection antibody and the signals for each sensor are rapidly rising. By $7 \mathrm{~min}$, the reaction has reached equilibrium and the signals from each sensor have begun to plateau. In this assay, CEA was present at the highest concentration and accordingly rose fastest, producing the largest signal. Lactoferin (LTF) was present at the next to highest concentration giving moderate signal while vascular endothelial growth factor (VEGF) was present at a low concentration. In addition, the epoxy coated sensors and BSA negative control sensors remained flat, indicating low electronic noise and negligible non-specific binding respectively. Furthermore, it is clear that each replica sensor in this assay gave approximately the same signal across the chip demonstrating the sensor to sensor reproducibility and the reproducibility of the biochemistry.

In these series of experiments, we have shown that by labeling antibodies with MNP tags, GMR biosensors are capable of exceptionally sensitive and selective multiplex protein detection in a single reaction well of only $20-50 \mu \mathrm{L}$. The combination of small sample volumes, high sensitivity and high multiplex capability establishes GMR biosensor arrays as one of the most compelling biosensor platforms for both basic science research and clinical medicine applications.

Lastly, Table 1 compares this work against prior art on GMR biosensing. Comparison of other biosensors can be found elsewhere (Tamanaha et al., 2008). A figure of merit (FOM) was created to capture the minimum number of detectable magnetic tags, the volume of the tags, and the sensor active area (Eq. (3)). This FOM does not differentiate the composition of the magnetic tags which may have different magnetic moments or if the tag is only partially magnetic (such as clusters of particles embedded in a matrix). Ideally, the FOM would quantify the magnetic moment per coverage area, but this requires information about the tags that is sometimes not available with commercial offerings. The FOM does, however, give a general view of the relative sensitivity of the system comprised of a sensor, magnetic tag, and electronics. It is prudent to note that achieving the lowest FOM is not necessarily beneficial if it does not translate into a lower biological limit of detection. While the values listed for biological limit of detection are not directly comparable between DNA and protein assays, it provides a relative comparison and roughly correlates with the FOM.

$\mathrm{FOM}=\frac{\text { volume } \times \text { number of tags }}{\text { sensor active area }}$

This work achieved an FOM of $4.4 \times 10^{-11}$ while also carrying this system sensitivity into a low biological limit of detection. Furthermore, the readout time of our system allows us to study binding and release kinetics of antibody-antigen interactions.

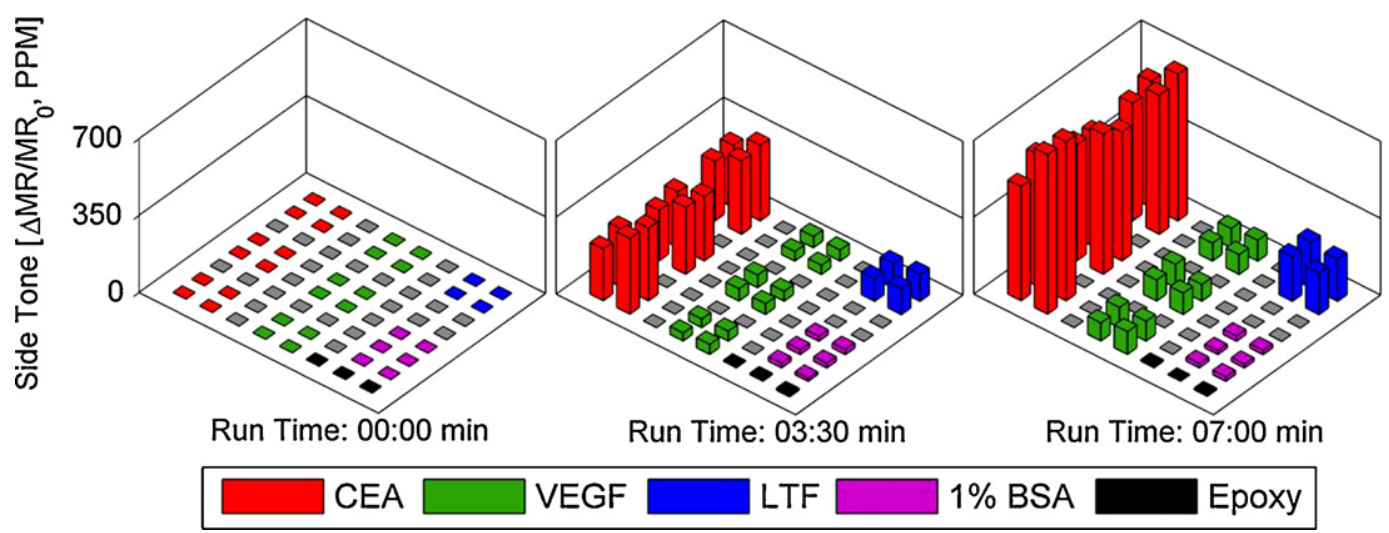

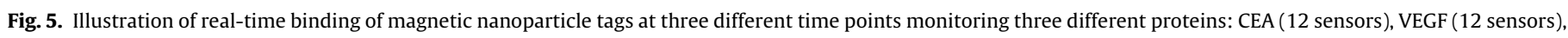
and LTF (4 sensors), and two negative controls: epoxy (3 sensors), and BSA (6 sensors). Real-time movie available online. 
Table 1

Summary of GMR biosensor systems.

\begin{tabular}{|c|c|c|c|c|c|}
\hline Research group & $\begin{array}{l}\text { Sensors } \\
\text { (\#/Chip) }\end{array}$ & $\begin{array}{l}\text { Readout time } \\
\text { (s/sensor) }\end{array}$ & $\begin{array}{l}\text { Magnetic tag } \\
\text { diameter (nm) }\end{array}$ & $\begin{array}{l}\text { Biological limit of } \\
\text { detection (fM) }\end{array}$ & $\operatorname{FOM}\left(\mathrm{m}^{3} / \mathrm{m}^{2}\right)$ \\
\hline Freitas (Martins et al., 2009) & 32 & - & 250 & 1 (DNA) & - \\
\hline Phillips (Dittmer et al., 2008) & 4 & 4.25 (est) & 500 & 800 (Protein) & $1.3 \times 10^{-10}$ \\
\hline Reiss (Reiss et al., 2005) & 256 & - & 860 & 25,000 (DNA) & - \\
\hline Tondra and Porter (Millen et al., 2008) & 5 & 24 & 1050 & - & $2.2 \times 10^{-8}$ \\
\hline Wang (Xu et al., 2008) & 4 & 1 & 50 & $10,000(\mathrm{DNA})$ & - \\
\hline Whitman (Rife et al., 2003) & 64 & 25 & 3300 & - & $1.5 \times 10^{-9}$ \\
\hline Wang (This work) & 64 & 0.06 & 50 & 5 (Protein) & $4.4 \times 10^{-11}$ \\
\hline
\end{tabular}

\section{Conclusion}

In summary, the circuit architecture presented demonstrates the viability of GMR sensors in biosensing by replacing the common Wheatstone bridge implementation with a TIA. Designed to reduce scan time while being easily scaled to large sensor arrays, the circuit implementation was thoroughly characterized. Subsequent analysis of noise and distortion shows that despite clear tradeoffs, the system in its entirety represents a solution that rivals many current assay techniques. Accordingly, a complete system-level optimization strategy was presented to determine the optimum bias and tickling fields for a particular magnetic tag and sensor pair, allowing for the sensitive detection of protein concentrations currently undetectable by ELISA. In the future we plan on using this system for studies of biomarker expression levels in cancer diagnostics and radiation triage.

\section{Acknowledgements}

This work was supported in part by National Cancer Institute Grants 1U54CA119367, National Science Foundation (NSF) Grant ECCS-0801385-000, Defense Threat Reduction Agency Grant HDTRA1-07-1-0030-P00005, Defense Advanced Research Projects Agency/Navy Grant N00014-02-1-0807 and the National Semiconductor Corporation. R.S.G. acknowledges financial support from Stanford Medical School MSTP program and an NSF graduate research fellowship.

\section{Appendix A. Supplementary data}

Supplementary data associated with this article can be found, in the online version, at doi:10.1016/j.bios.2010.01.038.

\section{References}

Baselt, D.R., Lee, G.U., Natesan, M., Metzger, S.W., Sheehan, P.E., Colton, R.J., 1998. Biosensors and Bioelectronics 13, 731-739. de Boer, B., Kahlman, J., Jansen, T., Duric, H., Veen, J., 2007. Biosensors and Bioelectronics 22, 2366-2370.

Daughton, J., Chen, Y., 1993. IEEE Transactions on Magnetics 29, 2705-2710.

Dittmer, W., de Kievit, P., Prins, M., Vissers, J., Mersch, M., Martens, M., 2008. Journal of Immunological Methods 338, 40-46.

Edelstein, R.L., Tamanaha, C.R., Sheehan, P.E., Miller, M.M., Baselt, D.R., Whitman, L.J., Colton, R.J., 2000. Biosensors and Bioelectronics 14, 805-813.

Ferreira, H.A., Feliciano, N., Graham, D.L., Freitas, P.P., 2005. Proceedings of the 49th Annual Conference on Magnetism and Magnetic Materials. AIP, Jacksonville, FL (USA), pp. 10Q904-13Q

Ferreira, H.A., Graham, D.L., Freitas, P.P., Cabral, J.M.S., 2003. Journal of Applied Physics 93, 7281-7286.

Gaster, R.S., Hall, D.A., Nielsen, C.H., Osterfeld, S.J., Yu, H., Mach, K.E., Wilson, R.J., Murmann, B., Liao, J.C., Gambhir, S.S., Wang, S.X., 2009. Nature Medicine 15, 1327-1332.

Hall, D.A., Gaster, R.S., Osterfeld, S.J., Murmann, B., Wang, S.X., in press. GMR biosensor arrays: correction techniques for reproducibility and enhanced sensitivity. Biosensors and Bioelectronics.

Han, S., Yu, H., Murmann, B., Pourmand, N., Wang, S.X., 2007. Solid-State Circuits Conference, 2007. ISSCC 2007. Digest of Technical Papers. IEEE International, pp. 168-594.

Koets, M., van der Wijk, T., van Eemeren, J., van Amerongen, A., Prins, M., 2009. Biosensors and Bioelectronics 24, 1893-1898.

Lenssen, K.H., Kuiper, A.E.T., van den Broek, J.J., van der Rijt, R.A.F., van Loon, A., 2000. Journal of Applied Physics AIP, 6665-6667.

Li, G., Wang, S.X., 2003. IEEE Transactions on Magnetics 39, 3313-3315.

Makinwa, K., Pertijs, M., van der Meer, J., Huijsing, J., 2007. Solid State Circuits Conference, 2007. ESSCIRC 2007. 33rd European, pp. 76-82.

Martins, V., Cardoso, F., Germano, J., Cardoso, S., Sousa, L., Piedade, M., Freitas, P., Fonseca, L., 2009. Biosensors and Bioelectronics 24, 2690-2695.

Millen, R.L., Nordling, J., Bullen, H.A., Porter, M.D., Tondra, M., Granger, M.C., 2008. Giant magenetoresistive sensors. 2. Detection of biorecognition events at self-referencing and magnetically tagged arrays. Analytical Chemistry 80, 7940-7946.

Osterfeld, S.J., Yu, H., Gaster, R.S., Caramuta, S., Xu, L., Han, S., Hall, D.A., Wilson, R.J., Sun, S., White, R.L., Davis, R.W., Pourmand, N., Wang, S.X., 2008. Proceedings of the National Academy of Sciences 105, 20637-20640.

Reiss, G., Brueckl, H., Huetten, A., Schotter, J., Brzeska, M., Panhorst, M., Sudfeld, D., Becker, A., Kamp, P.B., Puehler, A., Wojczykowski, K., Jutzi, P., 2005. Journal of Materials Research 20, 3294-3302.

Rife, J.C., Miller, M.M., Sheehan, P.E., Tamanaha, C.R., Tondra, M., Whitman, L.J., 2003. Sensors and Actuators A: Physical 107, 209-218.

Tamanaha, C., Mulvaney, S., Rife, J., Whitman, L., 2008. Biosensors and Bioelectronics 24, 1-13.

Xu, L., Yu, H., Akhras, M.S., Han, S., Osterfeld, S., White, R.L., Pourmand, N., Wang, S.X., 2008. Biosensors and Bioelectronics 24, 99-103. 